\title{
Prognostic value of the c-reactive protein/prognostic nutritional index ratio after hip fracture surgery in the elderly population
}

\author{
Hanru Ren ${ }^{1, *}$, Lianghao $\mathrm{Wu}^{1, *}$, Wankun $\mathrm{Hu}^{1}$, Xiuzhang $\mathrm{Ye}^{1}$ and Baoqing $\mathrm{Yu}^{1}$ \\ ${ }^{1}$ Department of Orthopaedics, Shanghai Pudong Hospital, Fudan University, Pudong Medical Center, Shanghai, China \\ *These authors have contributed equally to this work \\ Correspondence to: Baoqing Yu, email: yubaoqing1@163.com \\ Keywords: C-reactive protein/prognostic nutritional index, mortality, hip fracture, elderly population \\ Received: March 01, $2017 \quad$ Accepted: April 02, $2017 \quad$ Published: May 24, 2017 \\ Copyright: Ren et al. This is an open-access article distributed under the terms of the Creative Commons Attribution License 3.0 \\ (CC BY 3.0), which permits unrestricted use, distribution, and reproduction in any medium, provided the original author and source \\ are credited.
}

\section{ABSTRACT}

Background: More and more older patients receive the surgery after hip fracture. However, the mortality rate is high. Prognostic nutritional index (PNI) is associated with prognosis in hip fracture patients. In the current study, we proposed a novel prognostic score, named c-reactive protein/PNI ratio (CRP/PNI ratio), for predicting the prognosis for geriatric orthopedic population.

Methods: This is a prospective study. Eighty cases of hip fracture surgery in the elderly population were studied to reveal the relationship between the CRP / PNI ratio and the clinicopathological characteristics of the elderly patients. Clinical data included age, sex, weight, length of stay, duration of surgery, comorbidity, and biological data were collected. The primary endpoint was the 1-year mortality rate.

Results: Cox regression and log-rank tests were used to evaluate the correlation of CRP/PNI to the one-year mortality. The one-year mortality rate was low in the patients with a low CRP/PNI ratio $(P<0.001)$. Univariate and multivariate survival analyses proved that CRP/PNI was an important factor to predict the one-year mortality rate of the geriatric hip fracture surgery patients.

Conclusion: Low CRP/PNI ratio was significantly associated with low one-year mortality rate in older patients after hip fracture surgery.

\section{INTRODUCTION}

Hip fracture is a fracture of the upper part of the femur, and has high incidence in the elderly [1]. Hip fractures in aged people leads to high mortality, high risk of postoperative complication, and impaired quality of life [2-5]. The mortality is increased from $8.4 \%$ to $36 \%$ in the first postoperative year [6].

The risk factors for mortality following hip fracture surgery was demonstrated in some studies [7], we hope to reveal the clinically relevant biomarkers to evaluate prognosis and complication events. It has been reported that serum albumin level and lymphocyte count served as an independent prognostic factor in hip fracture patients [8]. The prognostic nutritional index (PNI) is calculated by the serum albumin concentration and the peripheral blood lymphocyte count [9]. Albumin is an indicator of nutritional status which is correlated with post-operative complications [10]. The PNI can be used to assess the immunological and nutritional status of surgery patients [11], and estimated according to the following formula: $10 \times$ serum albumin $(\mathrm{g} / \mathrm{dL})+0.05$ total lymphocyte count $\left(/ \mathrm{mm}^{3}\right)$ [12]. C-reactive protein (CRP) is an inflammatory marker which is closely related to the infections and outcomes in orthopaedics [13]. And PNI is associated with cancer mortality and has a prognostic value [14].

The correlation of CRP/PNI ratio and mortality after hip fracture surgery, especially in elderly population remains unknown. This study is aim to evaluate the predictive value of CRP/PNI ratio in elderly patients after orthopedic surgery, and compare it with clinical variables.

\section{RESULTS}

We estimated the CRP/PNI ratio in 80 patients, $35(43.75 \%)$ of whom were men and $45(56.25 \%)$ 
were women. The results are presented in Figure 1 and summarized in Table 1; the mean CRP and PNI were $11.3 \pm 9.6 \mathrm{mg} / \mathrm{L}$ and $41.3 \pm 11.0$, respectively. An inverse correlation was found between CRP and PNI with a correlation coefficient of -0.761 which was shown in Figure $1(P=0.016)$. The relationships between the CRP/ PNI ratio and clinical characteristics were shown in Table 1. Based on the receiver operating characteristics (ROC) analyses, the best cut-off value of $\mathrm{CRP} / \mathrm{PNI}$ ratio was 0.10 . Then, patients were divided into two groups: patients with $\mathrm{CRP} / \mathrm{PNI}$ ratio $\leq 0.10$ and patients with $\mathrm{CRP} / \mathrm{PNI}$ ratio $>0.10$. There were $42(52.5 \%)$ patients with $\mathrm{CRP} /$ PNI ratio $\leq 0.10$ and $38(47.5 \%)$ patients with $\mathrm{CRP} / \mathrm{PNI}$ ratio $>0.10$. CRP/PNI ratio is correlated significantly with CRP $(P<0.001)$, leukocytosis $(P=0.003)$, lymphocyte counts $(P<0.001)$, hypoalbuminemia $(P=0.001)$, PNI $(P$ $<0.001)$ and survival $(P<0.001)$, but no relationships were found between CRP/PNI ratio and other factors such as comorbidity, platelet count and anemia.

Concerning survival, only 3 of $42(7.1 \%)$ patients in the CRP/PNI ratio $\leq 0.10$ group died versus 38 of 38 $(100.0 \%)$ in the CRP/PNI ratio $>0.10$ group (Table 2$)$. The survival was significantly influenced by only CRP $(P$
$<0.001)$, lymphocyte counts $(P=0.013)$, hypoalbuminemia $(P=0.002)$, PNI $(P<0.001)$ and $\mathrm{CRP} / \mathrm{PNI}$ ratio $(P<0.001)$, while all variables were compared separately to survival status (Table 2). In univariate analysis, the Kaplan-Meier survival curves showed to no significant relationship between platelet count, leukocytosis, anemia and survival. The Kaplan-Meier survival curves of low CRP/PNI ratio versus high $\mathrm{CRP} / \mathrm{PNI}$ ratio showed a highly significant separation $(P<0.001$, Figure $2 \mathrm{C})$. Furthermore, patients with high CRP $(P<0.001$, Figure 2 A) or low PNI $(P<0.001$, Figure $2 \mathrm{~B})$ were also related to the poor 1-year survival, respectively. When a multivariate Cox proportional hazard model was constructed (including age, duration of surgery, weight, length of stay, sex, CRP, PNI, platelet count, leukocytosis, anemia, lymphocyte counts, hypoalbuminemia and CRP/PNI ratio), the CRP/PNI ratio was the strongest independent predictor of survival (Table 3 ).

The areas under the curve (AUC) was 0.998 for CRP/PNI ratio (95\% CI: 0.000-1.000, $P<0.001$ ), 0.928 (95\% CI: $0.861-0.990, P<0.001)$ for CRP and $0.953(95 \%$ CI: 0.002-0.996, $P<0.001)$ for PNI. Our results indicated that the CRP/PNI ratio was superior to the CRP or PNI (Figure 3 ) for the older patients after hip fracture surgery.

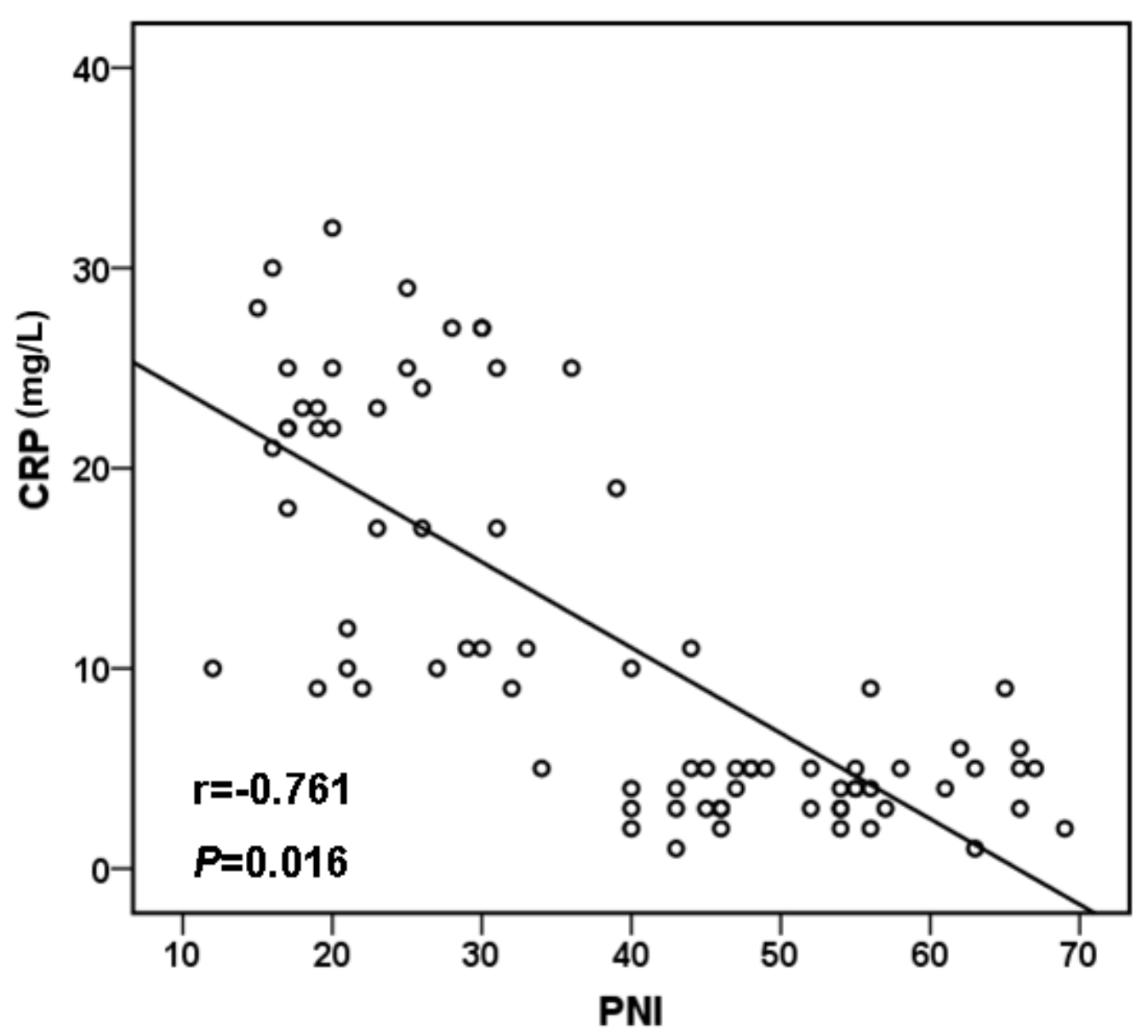

Figure 1: Pearson correlation. A signifcant negative correlation between CRP and PNI ( $\mathrm{r}=-0.761, P=0.016)$. CRP: C-reactive protein; PNI: prognostic nutritional index. 
Table 1: The CRP/PNI ratio and clinicopathological parameters

\begin{tabular}{|c|c|c|c|c|}
\hline & \multirow{2}{*}{ Case(n) } & \multicolumn{2}{|c|}{ CRP/PNI ratio } & \multirow{2}{*}{$p$ Value } \\
\hline & & $\leq \mathbf{0 . 1 0}$ & $>0.10$ & \\
\hline Age (years) & $86 \pm 5$ & $85 \pm 7$ & $87 \pm 3$ & 0.192 \\
\hline Men & 35 & 18 & 17 & 0.301 \\
\hline Weight (kg) & $62(49-78)$ & $61(45-74)$ & $63(55-78)$ & 0.762 \\
\hline Length of stay (day) & $7(5-14)$ & $6(5-10)$ & $9(6-14)$ & 0.162 \\
\hline $\begin{array}{l}\text { Duration of surgery } \\
\text { (min) }\end{array}$ & $140(111-170)$ & $140(111-170)$ & $140(111-170)$ & 0.912 \\
\hline \multicolumn{5}{|l|}{ Comorbidity } \\
\hline Diabetes mellitus & 34 & 17 & 17 & 0.684 \\
\hline $\begin{array}{l}\text { Cardiovascular } \\
\text { disease }\end{array}$ & 24 & 13 & 11 & 0.726 \\
\hline Hypertension & 63 & 32 & 31 & 0.710 \\
\hline Renal disefficiency & 21 & 12 & 9 & 0.691 \\
\hline \multicolumn{5}{|l|}{$\mathrm{CRP}, \mathrm{mg} / \mathrm{L}$} \\
\hline$<10$ & 43 & 39 & 4 & $0.000 *$ \\
\hline$\geq 10$ & 36 & 2 & 34 & \\
\hline \multicolumn{5}{|l|}{ Platelet count, $/ \mathrm{mm}^{3}$} \\
\hline$<214$ & 43 & 18 & 25 & 0.067 \\
\hline$\geq 214$ & 37 & 24 & 13 & \\
\hline \multicolumn{5}{|l|}{ Leukocytosis } \\
\hline Yes & 26 & 9 & 17 & $0.033 *$ \\
\hline No & 54 & 33 & 21 & \\
\hline \multicolumn{5}{|l|}{ Anemia } \\
\hline Yes & 42 & 25 & 17 & 0.262 \\
\hline No & 38 & 17 & 21 & \\
\hline \multicolumn{5}{|l|}{$\begin{array}{l}\text { Lymphocyte counts, } \\
\mathrm{mm}^{3}\end{array}$} \\
\hline$<1673$ & 43 & 31 & 12 & $0.000^{*}$ \\
\hline$\geq 1673$ & 37 & 11 & 26 & \\
\hline \multicolumn{5}{|l|}{ Hypoalbuminemia } \\
\hline Yes & 28 & 26 & 2 & $0.001 *$ \\
\hline No & 52 & 16 & 36 & \\
\hline \multicolumn{5}{|l|}{ PNI } \\
\hline$<44$ & 39 & 1 & 38 & $0.000 *$ \\
\hline$\geq 44$ & 41 & 41 & 0 & \\
\hline \multicolumn{5}{|l|}{ Survival status } \\
\hline Dead & 41 & 3 & 38 & $0.000 *$ \\
\hline Alive & 39 & 39 & 0 & \\
\hline
\end{tabular}

CRP: C-reactive protein; PNI: prognostic nutritional index; ${ }^{*} P<0.05$ is considered significant. 
Table 2: Survival status and clinicopathological parameters in 80 specimens

\begin{tabular}{|c|c|c|c|c|}
\hline & \multirow{2}{*}{ Case(n) } & \multicolumn{2}{|c|}{ Survival status } & \multirow{2}{*}{$p$ Value } \\
\hline & & Dead $=41$ & Alive $=39$ & \\
\hline Age (years) & $86 \pm 5$ & $87 \pm 5$ & $85 \pm 4$ & 0.059 \\
\hline Men & 35 & 19 & 16 & 0.142 \\
\hline Weight (kg) & $62(49-78)$ & $60(49-72)$ & $63(50-78)$ & 0.699 \\
\hline Length of stay (day) & $7(5-14)$ & $8(6-14)$ & $7(5-12)$ & 0.734 \\
\hline $\begin{array}{l}\text { Duration of surgery } \\
(\min )\end{array}$ & $140(111-177)$ & $146(112-177)$ & $139(111-168)$ & 0.209 \\
\hline \multicolumn{5}{|l|}{ Comorbidity } \\
\hline Diabetes mellitus & 34 & 20 & 14 & 0.550 \\
\hline $\begin{array}{l}\text { Cardiovascular } \\
\text { disease }\end{array}$ & 24 & 13 & 11 & 0.673 \\
\hline Hypertension & 63 & 33 & 30 & 0.897 \\
\hline Renal disefficiency & 21 & 11 & 10 & 0.803 \\
\hline \multicolumn{5}{|l|}{ CRP, mg/L } \\
\hline$<10$ & 43 & 7 & 36 & $0.000 *$ \\
\hline$\geq 10$ & 36 & 34 & 2 & \\
\hline \multicolumn{5}{|l|}{ Platelet count, $/ \mathrm{mm}^{3}$} \\
\hline$<214$ & 43 & 26 & 17 & 0.116 \\
\hline$\geq 214$ & 37 & 15 & 22 & \\
\hline \multicolumn{5}{|l|}{ Leukocytosis } \\
\hline Yes & 26 & 17 & 9 & 0.098 \\
\hline No & 54 & 24 & 30 & \\
\hline \multicolumn{5}{|l|}{ Anemia } \\
\hline Yes & 42 & 18 & 24 & 0.125 \\
\hline No & 38 & 23 & 15 & \\
\hline \multicolumn{5}{|l|}{$\begin{array}{l}\text { Lymphocyte counts,/ } \\
\mathrm{mm}^{3}\end{array}$} \\
\hline$<1673$ & 43 & 13 & 30 & $0.013 *$ \\
\hline$\geq 1673$ & 37 & 28 & 9 & \\
\hline \multicolumn{5}{|l|}{ Hypoalbuminemia } \\
\hline Yes & 28 & 26 & 2 & $0.002 *$ \\
\hline No & 52 & 15 & 37 & \\
\hline \multicolumn{5}{|l|}{ PNI } \\
\hline$<44$ & 39 & 39 & 0 & $0.000 *$ \\
\hline$\geq 44$ & 42 & 2 & 30 & \\
\hline \multicolumn{5}{|l|}{ CRP/PNI } \\
\hline$\leq 0.10$ & 42 & 3 & 39 & $0.000 *$ \\
\hline$>0.10$ & 38 & 38 & 0 & \\
\hline
\end{tabular}

CRP: C-reactive protein; PNI: prognostic nutritional index; ${ }^{*} P<0.05$ is considered significant. 


\section{DISCUSSION}

Because of the poor prognosis of acute surgery in elderly patients, biomarkers in clinical management of these patients are important. In our study, CRP/PNI ratio was used as an independent prognostic factor for elderly patients after orthopedic surgery. And this is the first study which examined the prognostic value of CRP/PNI ratio for elderly patients after orthopedic surgery.

Infection or traumas has a strong relationship with inflammation. Previous data have shown that CRP is a sensitive and non-specific inflammatory marker of human infection or traumas [15]. CRP is proved to be very useful in diagnosis and functioned as a monitor of infections in orthopaedics [13]. A retrospective analysis conducted by Kim et al. [15] revealed that preoperative CRP was an independent risk factor for 1-year mortality after hip fracture surgery in the elderly. In our current research, these patients with low CRP levels $(\leq 10.0$ $\mathrm{mg} / \mathrm{L}$ ) had better survival than those with CRP $>10.0$ $\mathrm{mg} / \mathrm{L}(83.7 \%$ vs. $5.6 \%, P<0.001)$. Regrettably, in the multivariate analyses, there was no evidence to prove that CRP was an independent prognostic factor $(P$ $=0.054)$.

The PNI was designed to assess the immunologic and nutritional aspects of surgical patients [16], and calculated by the serum albumin concentration and the lymphocyte count in the peripheral blood [17]. Hypoalbuminemia served as a potential preoperative predictor of outcomes [18]. Lu et al. showed that a lower serum albumin level and total lymphocyte count were important risk factors to predict the one-year mortality of elderly patients with fracture [19]. However, few studies focused on PNI in elderly patients after hip fracture surgery. In our data, PNI was not an independent prognostic factor $(P=0.067)$.
As CRP and PNI are affected by various conditions, the CRP/PNI ratio could therefore reduce the potential bias. The prognostic value of CRP/PNI ratio is better than CRP or PNI in elderly patients after hip fracture surgery. In this study, the CRP/PNI ratio has been investigated to assess the outcomes of the elderly patients after hip fracture surgery. Patients with low CRP/PNI ratio had a longer median survival time than those with high CRP/PNI ratio $(88.6 \%$ vs. $0 \%, P<0.001)$. CRP/PNI ratio has been demonstrated as an important predictive factor of 1-year survival by multivariate analyses $(P=0.006)$. The $\mathrm{CRP} / \mathrm{PNI}$ ratio had not been investigated before in hip fracture surgery patients before, especially in elderly patients. This is the first time to elucidate that CRP/PNI ratio is a predictor of hip fracture surgery in elderly patients.

In our Cox regression model, multivariate analyses revealed that the CRP/PNI ratio was an independent prognostic factor. Our data showed that the AUC was higher in CRP/PNI ratio than CRP (0.928) or PNI (0.963) by ROC analyses. High levels of CRP/PNI ratio could help us to avoid adverse consequences. Patients will benefit from the CRP/PNI ratio, especially in the elderly patients.

Nevertheless, several limitations should be paid attention in this study. Firstly, this study is a retrospective and single-institution study. Secondly, the number of patients was not adequate $(\mathrm{n}=80)$. Thence, larger prospective studies need to be carried out to confirm these preliminary results.

In summary, current data indicate that CRP/PNI ratio is correlated with 1-year survival in elderly patients after hip fracture surgery. Based on these results, we believe that CRP/PNI ratio is a novel and useful predictive factor in elderly patients after hip fracture surgery.
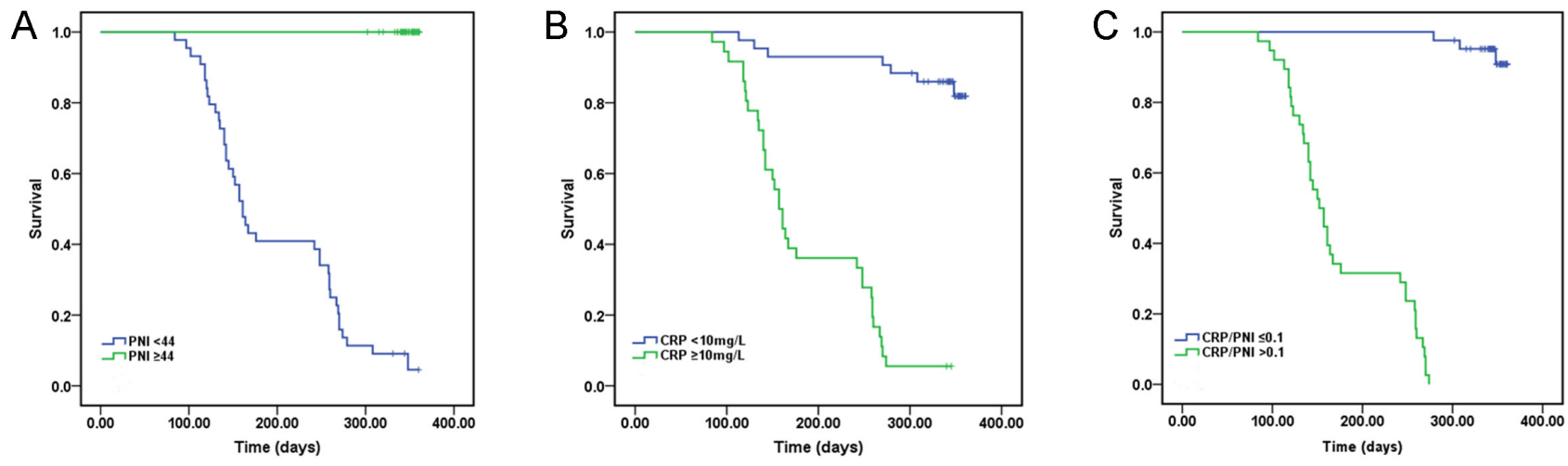

Figure 2: Kaplan-Meier survival curves stratifed by CPR, PNI and CRP/PNI ratio. (A and B) Patients with elevated CRP $(P$ $<0.001)$ or decreased PNI $(P<0.001)$ were associated with decreased 1 -year survival, respectively. (C) Patients with CRP/PNI ratio $\leq 0.10$ had a better 1-year survival than patients with CRP/PNI ratio $>0.10(P<0.001)$. CRP: C-reactive protein; PNI: prognostic nutritional index. 
Table 3: Contribution of various potential prognostic factors to survival by Cox regression analysis in 80 specimens

\begin{tabular}{lccc}
\hline & Hazard ratio & $\mathbf{9 5} \% \mathbf{C I}$ & $\boldsymbol{P}$ \\
\hline Age(years) & 1.5 & $0.775 \sim 2.430$ & 0.158 \\
Duration of surgery (min) & 0.7 & $0.341 \sim 3.776$ & 0.342 \\
Weight (kg) & 1.8 & $0.656 \sim 3.167$ & 0.782 \\
Length of stay (day) & 0.2 & $0.096 \sim 2.034$ & 0.053 \\
Sex & 1.9 & $0.127 \sim 3.907$ & 0.102 \\
CRP, mg/L & 3.3 & $1.337 \sim 8.737$ & 0.054 \\
PNI & 0.2 & $0.028 \sim 0.650$ & 0.067 \\
Platelet count, $/ \mathrm{mm}^{3}$ & 2.0 & $0.672 \sim 6.101$ & 0.210 \\
Leukocytosis & 0.9 & $0.313 \sim 2.860$ & 0.922 \\
Anemia & 1.3 & $0.432 \sim 3.811$ & 0.653 \\
Lymphocyte counts, $/ \mathrm{mm}^{3}$ & 2.1 & $1.093 \sim 6.107$ & 0.059 \\
Hypoalbuminemia & 1.4 & $0.897 \sim 3.357$ & 0.072 \\
CRP/PNI & 8.1 & $1.806 \sim 36.216$ & $0.006^{*}$ \\
\hline
\end{tabular}

CRP: C-reactive protein; PNI: prognostic nutritional index; CI: confidence interval; statistical analyses were performed by the log-rank test; $* P<0.05$ is considered significant.

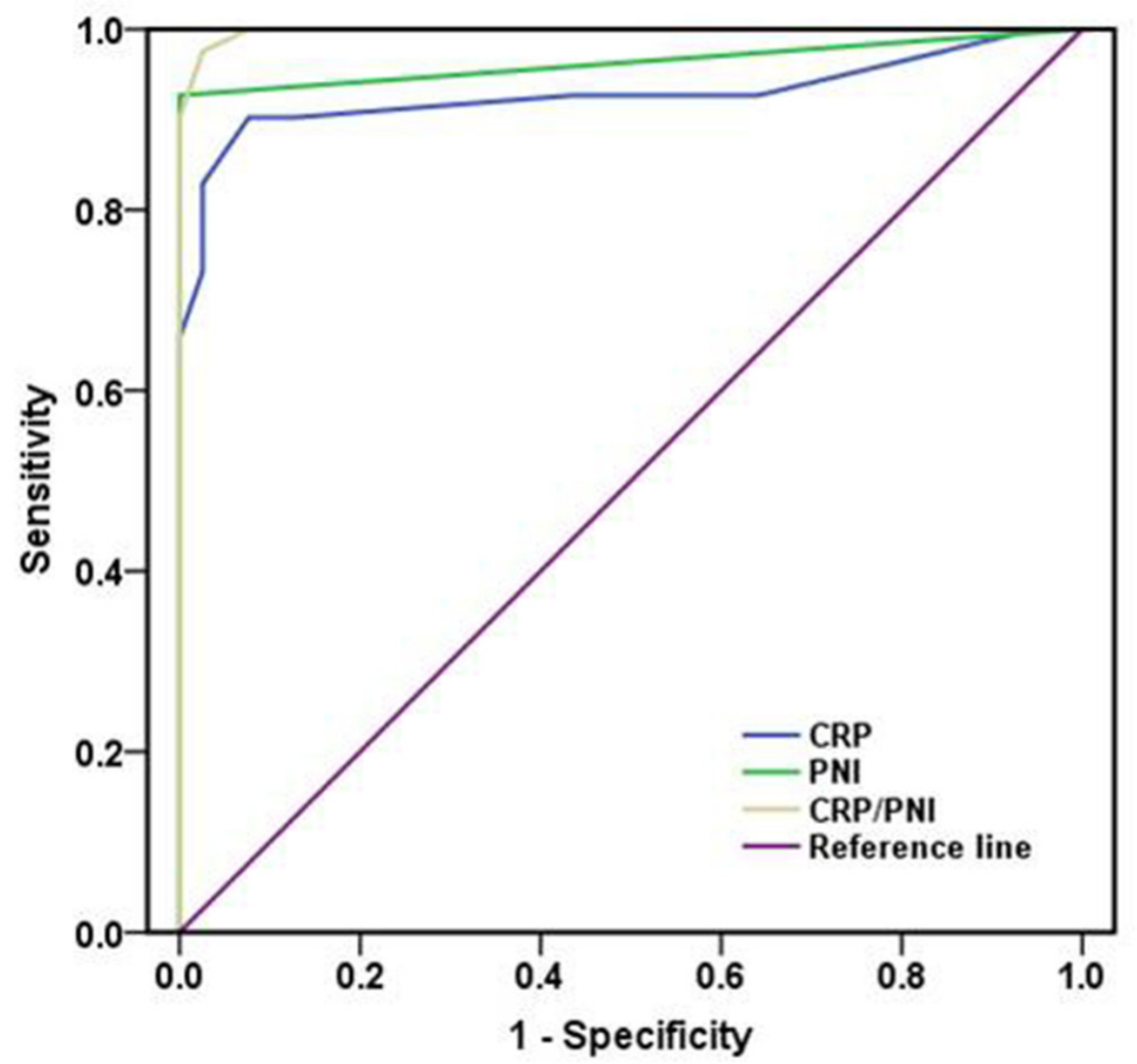

Figure 3: Comparison of the AUC for ROC curves. The AUC of the CRP/PNI ratio was higher than other CRP or PNI, indicating that the CRP/PNI ratio was superior to the CRP or PNI for prognosis. CRP: C-reactive protein; PNI: prognostic nutritional index; AUC: area under curve; ROC: receiver operating characteristics. 


\section{MATERIALS AND METHODS}

\section{Patients}

The data of 80 elderly patients after hip fracture surgery in Shanghai Pudong Hospital between 2015 and 2016 were collected in our study. The 70 years of age or older patients were included, and the patients were excluded if CRP, platelet count, leukocyte count, lymphocyte counts, hemoglobin and albumin measurements were lacking. Ethical approval was obtained for the study protocols and informed consents were obtained from each patient.

\section{Data collection}

We collected clinical data including age, sex, weight, length of stay, duration of surgery, comorbidity, biological data and survival time. The patients had been tested for serum CRP, platelet count, leukocyte count, lymphocyte counts, hemoglobin and albumin before surgery. At the same time, we carefully reviewed the associated comorbidities of these patients. The biological data were measured by automatic laser nephelometry (BN 100 analyzer, Germany). The normal values of CRP, platelet count, leukocyte count, lymphocyte counts, hemoglobin and albumin were $0-10 \mathrm{mg} / \mathrm{L}, 125-350$ $\times 10^{9} / \mathrm{L}, 3.5-9.5 \times 10^{9} / \mathrm{L}, 1.1-3.2 \times 10^{9} / \mathrm{L}, 110-175 \times 10^{9} / \mathrm{L}$, $35-50 \mathrm{~g} / \mathrm{L}$, respectively.

\section{Follow-up and endpoint}

The mean follow-up period for these patients was 8.7 months (range: 3-12 months). All patients were followed up by phone monthly. The endpoint was mortality within one year.

\section{Statistical analysis}

All statistical analyses were used the SPSS version 22.0 statistical software. The association between CRP and PNI were studied using the Spearman rank correlation test Survival analysis. For analysis of survival data, KaplanMeier curves were constructed, and the log-rank test was used for analysis. Univariate and multivariate analyses were performed using Cox's proportional hazards model. Differenceswere considered statistically significant when $\mathrm{P}$ value was less than 0.05 .

\section{ACKNOWLEDGMENTS}

This study was funded by the Outstanding Leaders Training Program of Pudong Health Bureau of Shanghai (grant PWR12013-01), the Program for Outstanding
Academic Leader of Shanghai, and the Program for Medical Key Department of Shanghai (Grant ZK 2015B17).

\section{CONFLICTS OF INTEREST}

The authors declare that they have no conflicts of interest.

\section{REFERENCES}

1. Heiberg KE, Bruun-Olsen V, Bergland A. The effects of habitual functional training on physical functioning in patients after hip fracture: the protocol of the HIPFRAC study. BMC Geriatr. 2017; 17: 23.

2. Katsoulis M, Benetou V, Karapetyan T, Feskanich D, Grodstein F, Pettersson-Kymmer U, Eriksson S, Wilsgaard T, Jorgensen L, Ahmed LA, Schottker B, Brenner H, Bellavia A, et al. Excess mortality after hip fracture in elderly persons from Europe and the USA: the CHANCES project. J Intern Med. 2017; 281: 300-10.

3. Mundi S, Pindiprolu B, Simunovic N, Bhandari M. Similar mortality rates in hip fracture patients over the past 31 years. Acta Orthop. 2014; 85: 54-9.

4. Huddleston JM, Whitford KJ. Medical care of elderly patients with hip fractures. Mayo Clin Proc. 2001; 76: 295-8.

5. Tarride JE, Burke N, Leslie WD, Morin SN, Adachi JD, Papaioannou A, Bessette L, Brown JP, Pericleous L, Muratov S, Hopkins RB. Loss of health related quality of life following low-trauma fractures in the elderly. BMC Geriatr. 2016; 16: 84.

6. Abrahamsen B, Van Staa T, Ariely R, Olson M, Cooper C. Excess mortality following hip fracture: a systematic epidemiological review. Osteoporos Int. 2009; 20: 1633-50.

7. Folbert EC, Hegeman JH, Gierveld R, Van Netten JJ, Velde DV, Ten Duis HJ, Slaets JP. Complications during hospitalization and risk factors in elderly patients with hip fracture following integrated orthogeriatric treatment. Arch Orthop Trauma Surg. 2017; 137: 507-15.

8. Symeonidis PD, Clark D. Assessment of malnutrition in hip fracture patients: effects on surgical delay, hospital stay and mortality. Acta Orthop Belg. 2006; 72: 420-7.

9. Nozoe T, Ninomiya M, Maeda T, Matsukuma A, Nakashima H, Ezaki T. Prognostic nutritional index: a tool to predict the biological aggressiveness of gastric carcinoma. Surg Today. 2010; 40: 440-3.

10. Adogwa O, Martin JR, Huang K, Verla T, Fatemi P, Thompson P, Cheng J, Kuchibhatla M, Lad SP, Bagley CA, Gottfried ON. Preoperative serum albumin level as a predictor of postoperative complication after spine fusion. Spine (Phila Pa 1976). 2014; 39: 1513-9. 
11. Han-Geurts IJ, Hop WC, Tran TC, Tilanus HW. Nutritional status as a risk factor in esophageal surgery. Dig Surg. 2006; 23: 159-63.

12. Hu Q, Wang G, Ren J, Ren H, Li G, Wu X, Gu G, Li R, Guo K, Deng Y, Li Y, Hong Z, Wu L, Li J. Preoperative prognostic nutritional index predicts postoperative surgical site infections in gastrointestinal fistula patients undergoing bowel resections. Medicine (Baltimore). 2016; 95: e4084.

13. Neumaier M, Braun KF, Sandmann G, Siebenlist S. C-reactive protein in orthopaedic surgery. Acta Chir Orthop Traumatol Cech. 2015; 82: 327-31.

14. Miao Y, Li S, Yan Q, Li B, Feng Y. Prognostic significance of preoperative prognostic nutritional index in epithelial ovarian cancer patients treated with platinum-based chemotherapy. Oncol Res Treat. 2016; 39: 712-9.

15. Kim BG, Lee YK, Park HP, Sohn HM, Oh AY, Jeon YT, $\mathrm{Koo} \mathrm{KH}$. C-reactive protein is an independent predictor for 1-year mortality in elderly patients undergoing hip fracture surgery: a retrospective analysis. Medicine (Baltimore). 2016; 95: e5152.

16. Buzby GP, Mullen JL, Matthews DC, Hobbs CL, Rosato EF. Prognostic nutritional index in gastrointestinal surgery. Am J Surg. 1980; 139: 160-7.

17. Onodera T, Goseki N, Kosaki G. [Prognostic nutritional index in gastrointestinal surgery of malnourished cancer patients]. Nihon Geka Gakkai Zasshi. 1984; 85: 1001-5.

18. Kamath AF, Nelson CL, Elkassabany N, Guo Z, Liu J. Low albumin is a risk factor for complications after revision total knee arthroplasty. J Knee Surg. 2017; 30: 269-75.

19. Lu J, Chen YY, Zhang L, Li YG, Wang C. Laboratory nutritional parameters predict one-year mortality in elderly patients with intertrochanteric fracture. Asia Pac J Clin Nutr. 2016; 25: 457-63. 